\title{
UTILIZACION DE ACEITE QUEMADO Y PARAQUAT EN LA DESECACIÓN DE SOYA Y SUS EFECTOS EN LA CALIDAD DE LA SEMILLA DURANTE EL ALMACENAMIENTO*
}

\author{
R. Ibañez**
}

\begin{abstract}
RESUMEN
El presente trabajo fue realizado en Pelotas, Río Grande do Sul Brasil, siendo su objetivo el de comparar el efecto del aceite lubricante quemado, con el paraquat aplicados durante la maduración fisiológica de las semillas de soya (Glicyne max L.) Merrill, variedad Bragg. Las dosis de aceite quemado utilizadas fueron de 5,3; 8,5 y 12,0 1/ha,se realizaron observaciones sobre la intensidad de desecación y perí odicamente fueron recolectadas vainas en cantidad de 30 por parcela. Con el propósito de darle seguimiento a la reducción del contenido de humedad de las semillas hasta llegar al $18 \%$, posteriormente esta humedad fue reducida a $10,5+0,5 \%$ y la semilla almacenada en cantidad de 2 kilos en bolsas de algodón por un período de 6 meses, bajo las condiciones ambientales del laboratorio del Centro de Entrenamiento de Semillas de la Universidad Federal de Pelotas. Las semillas secas fueron sometidas a la prueba de germinación, vigor y calidad sanitaria los tres y seis meses de almacenamiento. En las condiciones de este trabajo, el análisis e interpretación de los resultados permitieron concluir que, las dosis de 12,0 1/ha de aceite quemado y 2,0 1 de paraquat, aplicados al momento de la maduración fisiológica de las semillas, actuaron como desecantes de las plantas de soya, propician la obtención de semillas de mejor viabilidad y vigor, después de 6 meses de alamacenamiento, y garantizan mejor calidad sanitaria de las semillas.
\end{abstract}

\begin{abstract}
The assay was conducted in Pelotas, Rio Grande do Sul-Brasil, to compare the effect of used engine oil with to paraquat (Gramoxone) applied during the physiological maturition of soybean seeds (Glicyne $\max ($ L.) Merrill), Bragg variety. The oil doses were 5.3, 8.5 and $12 \mathrm{l} / \mathrm{ha}$. The desiccation intensity was determined and 30 pods per plot were periodically collected to follow the humidity content of the seeds, to $18 \%$. The humidity was later reduced to $10.5+0.5$ and $2.0 \mathrm{~kg}$ of seeds were stored, in cotton bags,during 6 months under the environmental conditions at the Centro de Entrenamiento de Semillas of the Federal University of Pelotas. The seeds were subjected to germination, vigor and phytosanitary tests at harvest time and after 3 and 6 months of storage. Under the conditions of this trial, the doses of $12 \mathrm{l} / \mathrm{ha}$ of oil and 2 1/ha of paraquat: a) act as desiccants for soybean plants, b) favors the production of seeds with better viability and vigor after 6 months of storage and c) guarantied better sanitary seed quality after 6 months of storage.
\end{abstract}

\section{INTRODUCCIÓN}

El uso de semillas de alta calidad en el cultivo de soya es uno de los principales factores que inciden en el mejoramiento de la productividad y calidad del grano cosechado.

La desecación en escala comercial en Brasil, está siendo utilizada en cultivos de arroz, caña de azúcar, papa, maíz y soya, Fundación de Ciencia y Tecnología (1985) y Pereira, Honda y Sartoni (1976). Los productos más utilizados en la desecación de las plantas son el Diquat, (Reglone) y el Paraquat (Gramoxone), siendo éste último el de mayor uso debido a su mayor espectro de acción sobre las malezas (Brasil 1979).
Los productores de semilla de soya en Brasil efectuán la desecación química de las plantas a partir del punto de madurez fisiológica, lo cual, al acelerar el proceso de secado de semillas, propicia una anticipación del período normal de cosecha, permitiendo así una serie de ventajas, que van desde la facilidad y eficiencia de cosecha hasta la obtención de una semilla de mejor calidad física, fisiológica y sanitaria (Pereira, Honda y Sartoni, 1976).

Sin embargo, con el propósito de obtener las ventajas deseadas, es indispensable el fiel cumplimiento de las exigencias y restricciones que se establecen en el uso de cualquier producto herbicida desecante, las cuales se justifican por los riesgos de contaminación de los granos con residuos tóxicos por encima de los permitidos por la

\footnotetext{
* Trabajo presentado a la XXXVII Reunión Anual del PCCMCA, Panamá. 1991.

** Comité Nacional de Semillas, Secretarí a Técnica. Ministerio de Desarrollo Agropecuario, Panamá.
} 
legislación local, al igual que los riesgos de intoxicación de los operadores, animales y contaminación ambiental. (Almeida et al 1986).

El aceite lubricante quemado, ha brindado resultados promisorios en la la protección de la semilla en condiciones adversas de humedad y facilitando su almacenamiento (Amorín 1985; Fetschenko, 1985; Peske, 1983; Delouche, 1985).su estudio para la obtención fisiológica, de semillas de mejor calidad, debe ser evaluado.

El presente trabajo se realizó con el objetivo de determinar los efectos sobre la calidad de la semilla de soya del aceite lubricante quemado, en diferentes dosis, en comparación con el herbicida desecante paraquat.

\section{MATERIALES}

Esta investigación se realizo durante el año 1986, en el campo experimental del Centro de Investigación Agropecuaria de Tierras Bajas de Clima Templado (CPATB/EMBRAPA), en Pelotas, Río Grande do Sul, Brasil. En lo referente a las pruebas de laboratorio, se utilizó el Laboratorio Didáctico de Análisis de Semillas, del Centro de Entrenamiento en Semillas de la Universidad Federal de Pelotas y en el Laboratorio de Fitopatología de CPATB/EMBRAPA.

La siembra se realizó con semilla fiscalizada de la variedad Bragg, suministrada por el Servicio de Producción de Semillas Básicas de EMBRAP A, el 7 de noviembre de 1985, la parcela consistió de cuatro hileras espaciales a $0,6 \mathrm{~m}$ con 10 semillas por metro lineal. Las prácticas agronómicas se realizaron de acuerdo a las recomendaciones técnicas para el cultivo.

Los tratamientos fueron ubicados dentro de un diseño de bloques completamente al azar, con cuatro repeticiones. Los tratamientos fueron: Paraquat (Gramoxone) 2,0 1/ha. Aceite quemado 5,3 1/ha. Aceite quemado 8,5 1/ha. Aceite quemado 12,0 1/ha. Testigo.

El paraquat fue aplicado con una bomba de mochila manual de espalda PV. marca Jacto, con boquillas de pulverización JD 14 y calibrada para un volumen de 165 1/ha; se utilizó un producto adherente.
El aceite quemado fue aplicado con una bomba de motor de espalda marca HASUTA, modelo BM-15. La dois de 5,3; 8,5 y 12,0 1/ha fueron obtenidas variando el tamaño del diámetro del orificio de los discos dosificadores. El aceite no fue filtrado, se usó tal como salió del carter del motor. La determinación del momento adecuado para la aplicación (madurez fisiológica) se hizo con base a la caracterización definitiva de la coloración de las semillas y del color del hilum, siendo el contenido de humedad de las semillas de $59 \%$.

\section{Evaluaciones de campo}

Con el propósito de verificar los efectos de los tratamientos sobre las plantas, se realizaron observaciones visuales después de la aplicación, se registró la intensidad de la caída de las hojas al cabo de una semana. Igualmente, se realizaron determinaciones periódicas del contenido de humedad de las semillas, y así medir los efectos en la velocidad de reducción de la humedad. Se utilizó el método de la estufa para dicha determinación.

La cosecha de las parcelas se definió cuando las semillas alcanzaron una humedad en torno a $18 \%$. Las vainas fueron acondicionadas en sacos de algodón y llevadas a un secador estacionario de fondo falso perforado, del Laboratorio Didáctico de Beneficiamiento de Semillas $\left(40^{\circ} \mathrm{C} / 15\right.$ horas), luego se procedió al desgrane, pre-limpieza y homogenización del tamaño en forma artesanal.

Finalmente, en caso necesario, se procedió al secado adicional de las semillas para llevadas a $10 \%$ de humedad y así, almacenadas por espacio de seis meses bajo las condiciones ambientales del Laboratorio de Análisis.

\section{Evaluaciones de laboratorio}

Las pruebas realizadas después de la cosecha y a los 3 y 6 meses de almacenamiento fueron:

1. Germinación 2.Vigor- Primer recuento Envejecimiento acelerado 3. Sanidad

Para el análisis estadístico de los resultados se utilizó el diseño en parcelas sub-divididas, donde los tratamientos constituyen las parcelas y los períodos de almacenamiento las sub-parcelas. Los valores fueron previamente transformados. 


\section{RESULTADOS Y DISCUSIÓN}

\section{Datos meteorológicos}

Los datos diarios de precipitación, temperatura y humedad relativa ocurridos en el período comprendido desde el momepto de la aplicación de los productos hasta la cosecha de las parcelas, aparecen en el Cuadro 1. Se puede observar que en el mismo día de la aplicación y en los siguientes, ocurrieron lluvias intensas y alta humedad relativa. La precipitación promedio en ese período fue de $122,7 \mathrm{~mm}$; la temperatura promedio de $20,2^{\circ} \mathrm{C}$ y humedad relativa de $92,0 \%$.

Cuadro 1. Precipitación, temperatura promedio y humedad relativa del aire diaria

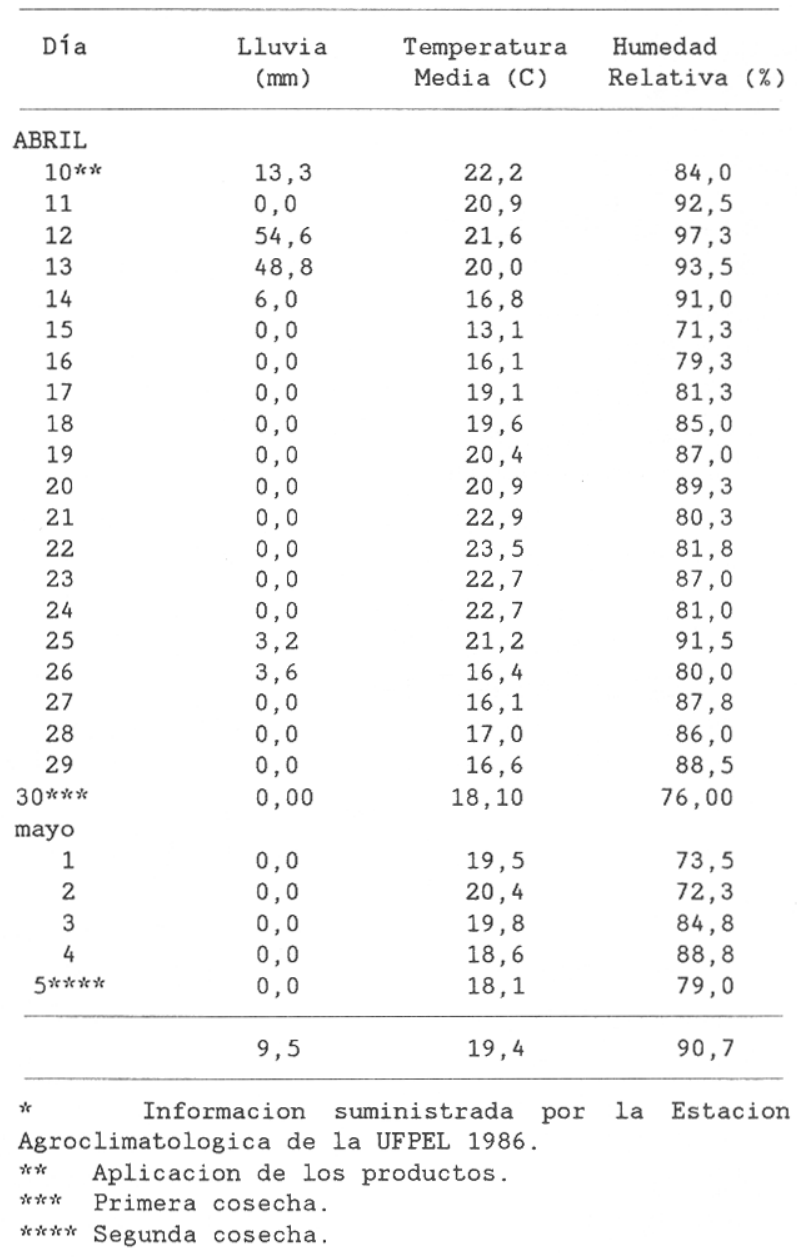

\section{Desecación de las plantas}

Los efectos de los tratamientos sobre la desecación y caída de las hojas una semana después de la aplicación, aparecen en el Cuadro 2.
Cuadro 2. Efectos de los tratamientos sobre la desecación y caída de las hojas una semana después de la aplicación. Pelotas, Rio Grande do Sul.

\begin{tabular}{|c|c|c|c|c|c|}
\hline \multicolumn{6}{|c|}{ Intensidad de Caida de Hojas } \\
\hline Tratamientos & $I$ & II & III & IV & $\mathrm{X}$ \\
\hline Paraquat & 5 & 5 & 5 & 4 & 4,8 \\
\hline Aceite quemado (12 1/ha) & 5 & 4 & 4 & 3 & 4,0 \\
\hline Aceite quemado $(8,51 /$ ha $)$ & 3 & 3 & 3 & 3 & 3,0 \\
\hline Aceite quemado $(5,31 /$ ha $)$ & 3 & 3 & 3 & 2 & 2,8 \\
\hline Testigo & 2 & 3 & 3 & 2 & 2,5 \\
\hline Promedio & 3,6 & 3,6 & 3,8 & 2,8 & \\
\hline Escala & $\begin{array}{l}\text { Prese } \\
\text { 1- Mu } \\
\text { 2- Re } \\
\text { 3- Po } \\
\text { 4- Ca } \\
\text { 5- } \mathrm{Au}\end{array}$ & $\begin{array}{l}\text { cia } \\
\text { has } \\
\text { ular } \\
\text { a } \\
\text { i na } \\
\text { enci }\end{array}$ & $\begin{array}{l}\text { n la } \\
\text { ojas } \\
\text { Cantic } \\
\text { a de ho }\end{array}$ & $\begin{array}{l}\text { anta } \\
\text { as }\end{array}$ & \\
\hline
\end{tabular}

\section{Reducción de la humedad}

En el Cuadro 3, se presentan los porcentajes promedio de humedad de las semillas después de la aplicación de los productos, la fecha y humedad de cosecha y el intervalo entre aplicación y cosecha. No hubo prácticamente ningún efecto de los tratamientos en la reducción de la humedad al cabo de cinco días, considerándose como causa principal la alta humedad relativa (Cuadro 1).

El análisis estadístico de los resultados reveló diferencias al nivel de $1 \%$ de probabilidad para los tratamientos, épocas e interacción.

El uso del paraquat y la dosis mayor de aceite quemado anticiparon la cosecha en cinco días con relación a los otros tratamientos, principalmente en comparación con el testigo, siendo su efecto en la reducción del contenido de humedad de las semillas, sobre todo en el período comprendido entre 5 y 8 días después de la aplicación, bastante semejante al obtenido en otras investigaciones, en un rango de humedad de las semillas entre 50 y $60 \%$.

Análisis de calidad

El análisis de varianza del porcentaje de germinación reveló diferencia significativa al 5\% de probabilidad para los tratamientos y épocas de almacenamiento. Los resultados de germinación se presentan en el Cuadro 4.

Se puede observar luego de 6 meses de almacenamiento, que las semillas provenientes de las parcelas tratadas con paraquat y dosis mayor de aceite 
quemado se mostraron significativamente superiores al testigo, con germinaciones satisfactorias de $86 \%$, en comparación con los otros tratamientos que no presentaron la germinación mínima exigida para la comercialización de semillas, o sea $80 \%$. Igualmente, el promedio de los tres períodos de almacenamiento, ambos tratamientos mantuvieron los mayores porcentajes de germinación $(\mathrm{P}<=0,05)$. efecto significativo al $(\mathrm{P}<=0,01$ para tratamiento $\mathrm{y}$ épocas de almacenamiento (Cuadro 5).

Se puede observar que al cabo de 6 meses de almacenamiento, las semillas presentaron una gran reducción en el vigor, siendo las mayores reducciones en las semillas provenientes de los tratamientos con las dosis media y menor de aceite quemado y del testigo. El

Cuadro 3. Porcentaje promedio de humedad de las semillas después de la aplicación de los tratamiento humedad y fecha de cosecha e intervalo entre aplicación y cosecha. Pelotas, Rio Grande do Sul, 1986.

\begin{tabular}{cccccc}
\hline & \multicolumn{5}{c}{ Tratamientos } \\
\cline { 2 - 6 } $\begin{array}{c}\text { Dias despues } \\
\text { de Aplicacion }\end{array}$ & Paraquat & $\begin{array}{c}\text { Aceite } \\
\text { Quemado } \\
(12,01)\end{array}$ & $\begin{array}{c}\text { Aceite } \\
\text { Quemado } \\
(8,51 \mathrm{l})\end{array}$ & $\begin{array}{c}\text { Aceite } \\
\text { Quemado } \\
(5,3 \mathrm{lt})\end{array}$ & Testigo \\
\hline 0 & $59,1 \mathrm{a}$ & $59,1 \mathrm{a}$ & $59,1 \mathrm{a}$ & $59,1 \mathrm{a}$ & $59,1 \mathrm{a}$ \\
5 & $57,2 \mathrm{a}$ & $57,4 \mathrm{a}$ & $57,5 \mathrm{a}$ & $58,6 \mathrm{a}$ & $58,6 \mathrm{a}$ \\
8 & $35,2 \mathrm{~d}$ & $37,5 \mathrm{c}$ & $41,5 \mathrm{~b}$ & $46,2 \mathrm{a}$ & $45,8 \mathrm{a}$ \\
15 & $22,3 \mathrm{c}$ & $22,8 \mathrm{c}$ & $24,5 \mathrm{bc}$ & $25,8 \mathrm{ab}$ & $27,6 \mathrm{a}$ \\
20 & $17,8 \mathrm{~d}$ & $18,6 \mathrm{~cd}$ & $20,9 \mathrm{bc}$ & $22,4 \mathrm{ab}$ & $23,5 \mathrm{a}$ \\
25 & ----- & ----- & 19,0 & 19,6 & 19,1 \\
Humedad de Cosecha & 17,8 & 18,6 & 19,0 & 19,6 & 19,1 \\
$\begin{array}{c}\text { Fecha de Cosecha } \\
\text { Aplicacion - Cosecha }\end{array}$ & $30 / 4 / 86$ & $30 / 4 / 86$ & $5 / 5 / 86$ & $5 / 5 / 86$ & $5 / 5 / 86$ \\
\hline
\end{tabular}

Cuadro 4. Germinación promedio (\%) de las semillas de soya en función de los tratamientos y periodos de almacenamiento. Pelotas, Rio Grande do Sul.

\begin{tabular}{|c|c|c|c|c|c|}
\hline & Tratamientos & $\frac{\text { Periodo }}{0}$ & $\frac{\text { Almac, }}{3}$ & $\frac{\text { (Meses) }}{6}$ & Promedio \\
\hline $\begin{array}{l}\text { Paraqua } \\
\text { Aceite } \\
\text { Aceite } \\
\text { Aceite } \\
\text { Testigo }\end{array}$ & $\begin{array}{l}\text { at } \\
\text { quemado } \\
\text { quemado } \\
\text { quemado }\left(\begin{array}{ll}12,0 & 1 / \mathrm{ha}) \\
8,5 & 1 / \mathrm{ha}) \\
5,3 & 1 / \mathrm{ha})\end{array}\right) \\
0\end{array}$ & $\begin{array}{l}88,0 \mathrm{a} \\
88,0 \mathrm{a} \\
82,0 \mathrm{ab} \\
82,0 \mathrm{ab} \\
80,0 \mathrm{~b}\end{array}$ & $\begin{array}{l}88,0 \mathrm{a} \\
87, \mathrm{Oa} \\
81, \mathrm{Oab} \\
75, \mathrm{Ob} \\
77, \mathrm{Ob}\end{array}$ & $\begin{array}{l}86,0 \mathrm{a} \\
86, \mathrm{Oa} \\
78, \mathrm{Obc} \\
76, \mathrm{Oc} \\
78, \mathrm{Obc}\end{array}$ & $\begin{array}{l}87,0 \mathrm{a} \\
87,0 \mathrm{a} \\
81,0 \mathrm{~b} \\
78,0 \mathrm{~b} \\
78,0 \mathrm{~b}\end{array}$ \\
\hline \multicolumn{2}{|r|}{ Promedio } & 84,0 & 82,0 & 81,0 & \\
\hline
\end{tabular}

Cuadro 5. Germinacion promedio (\%) de las semillas de soya, reveladas por la prueba de envejecimiento acelerado, su función de los tratamientos y periodos de almacenamiento.

\begin{tabular}{|c|c|c|c|c|c|}
\hline \multicolumn{2}{|r|}{ Tratamientos } & \multicolumn{2}{|c|}{ Periodo Almac, (Meses) } & $\frac{\text { (Meses) }}{6}$ & 2 Promedio \\
\hline $\begin{array}{l}\text { Paraqua } \\
\text { Aceite } \\
\text { Aceite } \\
\text { Aceite } \\
\text { Testigo }\end{array}$ & $\begin{array}{l}\text { at } \\
\text { quemado } \\
\text { quemado } \\
\text { quemado }\left(\begin{array}{lll}12,0 & 1 / \mathrm{ha}) \\
8,5 & 1 / \mathrm{ha}) \\
5,3 & 1 / \mathrm{ha}\end{array}\right) \\
0\end{array}$ & $\begin{array}{l}84,0 a \\
85,0 a \\
80,0 a \\
76,0 a \\
78,0 a\end{array}$ & $\begin{array}{l}85,0 \mathrm{a} \\
85,0 \mathrm{a} \\
77,0 \mathrm{ab} \\
73,0 \mathrm{~b} \\
73,0 \mathrm{~b}\end{array}$ & $\begin{array}{l}69,0 \mathrm{a} \\
66,0 \mathrm{ab} \\
51,0 \mathrm{c} \\
54,0 \mathrm{bc} \\
56,0 \mathrm{bc}\end{array}$ & $\begin{array}{l}80,0 \mathrm{a} \\
79,0 \mathrm{a} \\
70,0 \mathrm{~b} \\
68,0 \mathrm{~b} \\
69,0 \mathrm{~b}\end{array}$ \\
\hline & Promedio & 81,0 & 79,0 & 59,0 & \\
\hline
\end{tabular}

En relación con la prueba de envejecimiento acelerado para evaluar el vigor de las semillas se encontró paraquat fue significativamente superior a los demás tratamientos, pero no difirió de la dosis mayor de aceite quemado, en relación al vigor de las semillas. El promedio de los tres períodos de almacenamiento permitió obtener una significancia superior del paraquat y de la mayor dosis de aceite quemado.

Los niveles de infestación de Fusarium sp, Cercospora Kikuchi, Penicillium sp y Aspergillus sp.se muestran en los Cuadros 6 y 7. El análisis estadístico, para la incidencia de microorganismos en la semilla, reveló diferencia no significativa entre tratamientos, a excepción de Aspergillus sp. que fue significativa al $1 \%$ de probabilidad. En relación con las épocas de almacenamiento hubo diferencias al $5 \%$ de las variables Penicillium sp, Aspergillus sp. y Colletotrichum sp., siendo al $1 \%$ para el resto de los microorgamsmos.

La alta incidencia de los hongos Fusarium y Cercospora, tanto a los tres como a los seis meses de almacenamiento, estuvo relacionado con las condiciones climáticas prevalecientes durante el período de floración y maduración de las semillas. Sin embargo, su presencia no fue limitante en la obtención de resultados satisfactorios en la calidad fisiológica de las semillas. 
Cuadro 6. Porcentaje de incidencia de hongos en semilla de soya, después de 3 y 6 meses de almacenamiento. Pelotas, Rio Grande do Sul, 1986.

\begin{tabular}{|c|c|c|c|c|c|c|}
\hline \multirow[b]{2}{*}{ Tratamientos } & \multicolumn{2}{|c|}{ Fusarium sp. } & \multirow[b]{2}{*}{ Media } & \multicolumn{2}{|c|}{ Cercospora K. } & \multirow[b]{2}{*}{ Media } \\
\hline & 3 Meses & 6 Meses & & 3 Meses & 6 Meses & \\
\hline Paraquat & $68,8 \mathrm{~b}$ & $66,0 \mathrm{a}$ & $66,0 \mathrm{ab}$ & $63,3 a$ & $43,3 a$ & $52,0 \mathrm{a}$ \\
\hline Aceite quemado $(5,31 /$ ha $)$ & $84,3 a$ & $63,5 a$ & $73,4 \mathrm{ab}$ & $56,3 a$ & $35,5 b$ & $45,0 \mathrm{ab}$ \\
\hline Aceite quemado $(8,51 /$ ha $)$ & $83,0 \mathrm{a}$ & $60,3 a$ & $71,0 \mathrm{ab}$ & $54,0 \mathrm{ab}$ & $30,8 \mathrm{~b}$ & $41,7 b$ \\
\hline Aceite quemado $(12,01 /$ ha $)$ & $80,0 \mathrm{ab}$ & $62,0 \mathrm{a}$ & $70,6 \mathrm{ab}$ & $56,0 \mathrm{a}$ & $40,3 \mathrm{ab}$ & $47,5 \mathrm{ab}$ \\
\hline Testigo & $82,3 \mathrm{ab}$ & $72,0 \mathrm{a}$ & $76,9 a$ & $54,0 a$ & $45,5 a$ & $49,3 \mathrm{ab}$ \\
\hline Promedio & $79,8 \mathrm{~A}$ & $64,8 B$ & & $56,9 \mathrm{~A}$ & $39,0 B$ & \\
\hline
\end{tabular}

Cuadro 7. Porcentaje de incidencia de hongos en semilla de soya, después de 3 y 6 meses de almacenamiento. Pelotas, Rio Grande do Sul. 1986.

\begin{tabular}{|c|c|c|c|c|c|c|}
\hline \multirow[b]{2}{*}{ Tratamientos } & \multicolumn{3}{|c|}{ Penicillium spp } & \multicolumn{3}{|c|}{ Aspergillus spp. } \\
\hline & 3 Meses & 6 Meses & Media & 3 Meses & 6 Meses & Media \\
\hline Paraquat & $7.3 \mathrm{a}$ & $10.8 \mathrm{ab}$ & $8.5 a$ & $3.0 \mathrm{c}$ & $4.3 b$ & $3.5 c$ \\
\hline Aceite quemado ( $5.31 /$ ha $)$ & $17.0 \mathrm{a}$ & $18.5 \mathrm{ab}$ & $14.8 \mathrm{a}$ & $8.3 b$ & $15.5 a$ & $11.1 \mathrm{ab}$ \\
\hline Aceite quemado ( $8.51 /$ ha) & $13.8 \mathrm{a}$ & $20.8 \mathrm{a}$ & $16.3 a$ & $5.8 \mathrm{bc}$ & $13.0 \mathrm{a}$ & $8.9 \mathrm{ab}$ \\
\hline Aceite quemado ( $12.01 / \mathrm{ha})$ & $7.3 a$ & $8.8 \mathrm{~b}$ & $7.7 \mathrm{a}$ & $7.0 \mathrm{bc}$ & $6.5 b$ & $6.4 \mathrm{bc}$ \\
\hline Testigo & $12.8 \mathrm{a}$ & $18.3 \mathrm{ab}$ & $14.6 \mathrm{a}$ & $14.5 a$ & $14.5 a$ & $14.2 \mathrm{a}$ \\
\hline Promedio & $11.6 \mathrm{~A}$ & $14.9 \mathrm{~B}$ & & $7.7 \mathrm{~A}$ & $10.7 \mathrm{~B}$ & \\
\hline
\end{tabular}

\section{CONCLUSIONES}

La dosis de 12,0 1/ha de aceite quemado, y 2,0 1/ha de Paraquat, aplicados en el momento de la maduración fisiológica de las semillas, actuaron como desecante de las plantas de soya.

Las dosis de 12,0 1/ha de aceite quemado y 2,0 1/ha de Paraquat, aplicados en el momento de la maduración fisiológica, propician la obtención de semillas de mejor viabilidad y vigor, después de seis meses de almacenamiento.

Las dosis de 12,0 1/ha de aceite quemado y 2,0 1/ha de paraquat, tuvieron mejor calidad sanitaria de las semillas después de 6 meses, en lo que respecta a la ocurrencia de semillas sanas y hongos de almacenamiento.

\section{LITERATURA CITADA}

ALMEIDA, F.S; LEITE, C.RF. AGUILAR,AP.; RODRIGUEZ, B.N. 1986. Residuos de paraquat en granos de soya cuando es usado como desecan te del cultivo. In: Resultados de pesquisa de área de herbología, safras de 1984/85 y 1985/86. Londrina, 1APAR. pp.107-15.
AMORIN, RC. 1985. Efeite de protetores na germiniacao e emergencia de dese mentes de soya (Glycine max L). Dissertacao de mestrado. Pelotas, Universidade Federal de Pelotas. 66p.

SECRETARIA NACIONAL DE DEFENSA SANITARIA VEGETAL. BRASIL. 1979. Divisao de Produtos Fitosanitarios. Catálogo dos defensivos Agrícolas. Brasilia. $94 \mathrm{p}$.

FERSCHENKO, O. B. 1985. Protetores de sementes de soya em relacao ao armazenamento. Dissertacao de mestrado. Pelotas, Universidade Federal de Pelotas. 71 p.

FUNDACAO DE CIENCIA E TECNOLOGIA 1985. Projeto de pesquisa DEF GLOBAL II. Residuos de defensivos agrícolas em frutas, hortalizas, arroz, trigo, soja e graos importados. Relatorio parcial; soya. Brasil. Porto Alegre. 99p.

PEREIRA, J. L.; HONDA, A.I.; SARTORI, S. 1976. Efeitos da dessecacao pre-colheita nos graos de soja. In: Manual de dessecacao da soja.Sao Paulo, Compahnia Imperial de Ind. Qca do Brasil-Depto. Agricola. 16 p.

PESKE, S. 1983. Germination and emergence of soybean seed as related to moistura stree. Thesis $\mathrm{Ph}$. D. Mississippi, Mississipi Sta te Univ. 81 p.

PESKE,S.; DELOUCHE. J. C. 1985. Semeadura de soja em condicoes de baixa unidade do solo. Pesquisa Agropecuaria Brasileira 20 (1): 69-85. 